\title{
10-GHz MIXSEL: An Integrated Ultrafast Semiconductor Disk Laser with 2.2 W Average Power
}

\author{
V. J. Wittwer, B. Rudin, D. J. H. C. Maas, M. Hoffmann, O. D. Sieber, Y. Barbarin, M. Golling, T. Südmeyer, \\ U. Keller \\ Department of Physics, Institute of Quantum Electronics, ETH Zurich, Wolfgang-Pauli-Str. 16, 8093 Zurich, Switzerland. \\ vwittwer@phys.ethz.ch
}

\begin{abstract}
We present a 10-GHz Modelocked Integrated External-Cavity Surface Emitting Laser (MIXSEL) with $2.2 \mathrm{~W}$ average power in 21-ps pulses, which is the highest power level from any $10-\mathrm{GHz}$ modelocked semiconductor laser. (C)2011 Optical Society of America OCIS codes: (140.4050) Mode-locked lasers; (250.7260) Vertical cavity surface emitting lasers
\end{abstract}

\section{Introduction}

Compact high-power multi-GHz optical sources are of high interest for many applications, such as optical clocking, photonic switching or optical sampling. VECSELs (Vertical External Cavity Surface Emitting Lasers) modelocked with semiconductor saturable absorber mirrors (SESAMs) [1,2] achieve excellent beam quality, and high power operation at high repetition rates (486-fs pulses with $30 \mathrm{~mW}$ average power at $10 \mathrm{GHz}$ [3], $1.4 \mathrm{~W}$ in 6.1-ps pulses at $10 \mathrm{GHz}$ [4], and $50 \mathrm{GHz}$ with $102 \mathrm{~mW}$ [5]). However, the laser contains two separate semiconductor elements in a folded cavity, which is a challenge for cost-efficient high volume fabrication, as well as for reaching higher repetition rates. Modelocked integrated external-cavity surface emitting lasers (MIXSELs) [6, 7] combine gain and absorber in one semiconductor structure, enabling modelocking in a simple straight cavity and the possibility of a quasi-monolithic design [7]. Since the first MIXSEL demonstration in 2007 (40 mW in 35 ps pulses at 2.8 GHz) [6], we improved our MIXSEL design by introducing novel low saturation fluence quantum dot (QD) saturable absorbers. This allows for an antiresonant MIXSEL design with high growth tolerance. Additionally, thermal management was substantially improved by direct soldering the MIXSEL semiconductor chip onto a CVD diamond heat spreader and subsequent substrate removal. At $2.5 \mathrm{GHz}$ we recently demonstrated power scaling up to $6.4 \mathrm{~W}$ [8].

Initial results at $10 \mathrm{GHz}$ obtained with copper heat spreader limited the power to $190 \mathrm{~mW}$. Here we present a $10-\mathrm{GHz}$ MIXSEL with $2.2 \mathrm{~W}$ average power in 21-ps pulses, which is to our knowledge higher than any other $10-\mathrm{GHz}$ modelocked semiconductor laser. The MIXSEL consisted of a simple 15-mm long straight cavity with only two cavity elements, the MIXSEL chip and the external output coupler.

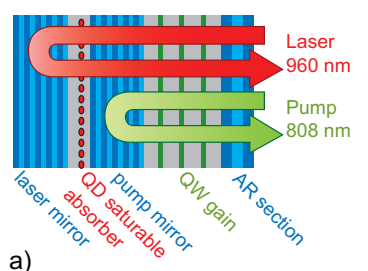

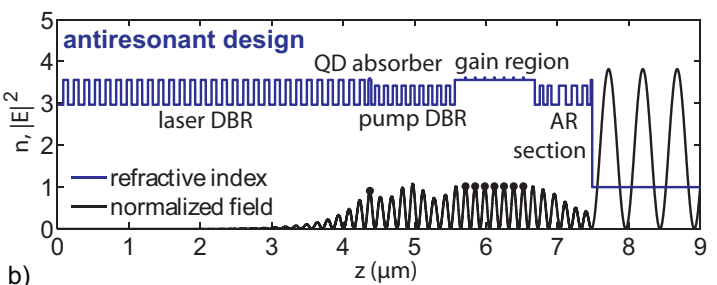

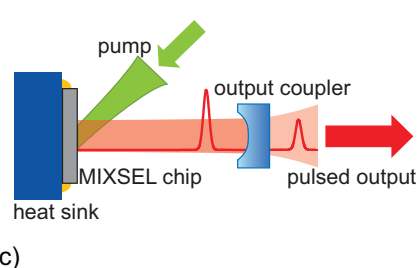

Fig. 1: MIXSEL concept (a); refractive index of semiconductor layer structure and standing wave intensity pattern (b); MIXSEL cavity (c).

\section{MIXSEL design and fabrication}

Figure la shows the concept of the MIXSEL. The refractive index profile (blue line) as well as the standing wave intensity pattern for the laser light at $960 \mathrm{~nm}$ (black line) are shown in Figure 1b. The structure is composed of a DBR for the laser light (30 pairs AlAs/GaAs), the quantum dot saturable absorber (one layer of self-assembled InAs quantum dots embedded in GaAs spacer layers), a DBR for the pump light (9 pairs $\mathrm{Al}_{0.2} \mathrm{Ga}_{0.8} \mathrm{As} / \mathrm{AlAs}$ ), the gain region ( 7 InGaAs quantum wells separated by GaAs spacer layers) and an anti-reflection section $\left(\mathrm{Al}_{0.2} \mathrm{Ga}_{0.8} \mathrm{As} / \mathrm{AlAs}\right)$. The intermediate mirror reduces pump light pre-saturation of the absorber.

The MIXSEL structure was grown by MBE in reverse order on a $600-\mu \mathrm{m}$ GaAs wafer in one single run. First etch stop layers were grown followed by the other sections. The chips are processed as follows: first small pieces are cleaved from the wafer, metalized and soldered to CVD diamond heat spreaders (thermal conductivity 
$>1800 \mathrm{~W} \cdot \mathrm{K}^{-1} \cdot \mathrm{m}^{-1}$ ) with a fluxless indium soldering process under vacuum. Then the GaAs substrate is removed by a chemical wet etching procedure. The reduced thickness of the semiconductor material leads to a low thermal impedance and to a nearly one-dimensional heat flow into the heat sink, which makes the device power scalable in spot size.

\section{Experiment and results}

The MIXSEL setup consists of a straight cavity created by the flat MIXSEL structure and a curved output coupler (Fig. 1c) with $1.5 \mathrm{~m}$ radius of curvature and $0.5 \%$ transmission. The cavity was $15 \mathrm{~mm}$ long to obtain $10 \mathrm{GHz}$ repetition rate (Fig. 2c). The heat sink temperature was controlled by a Peltier element to $-10^{\circ} \mathrm{C}$. The structure was pumped with a $100-\mathrm{W}$ fiber-coupled laser diode array at $808 \mathrm{~nm}$ at an angle of $45^{\circ}$. The pump spot radius was $215 \mu \mathrm{m}$ matched to the size of the laser mode on the MIXSEL chip, ensuring fundamental transverse mode operation. For a pump power of $25.4 \mathrm{~W}$ stable and self-starting modelocking was obtained with an average power of $2.2 \mathrm{~W}$. The optical-to-optical efficiency was $8.7 \%$. The pulse duration was $\approx 21 \mathrm{ps}$ (Fig. $2 \mathrm{a}$ ) and the center wavelength was $966 \mathrm{~nm}$ (Fig 2b). The rf-spectrum (Fig. 2c) shows side peaks with an offset frequency of $\approx 44 \mathrm{MHz}$. Most likely, these are caused by polarization instabilities, as they could be attenuated with a polarizer in the detection arm. Like in VCSELs, it should be possible to stabilize the polarization by introducing a surface grating [9] on our MIXSEL structure. Beam quality measurement at a slightly reduced output power of $1.2 \mathrm{~W}$ showed excellent $\mathrm{M}^{2}$ values of $<1.05$ in both directions.
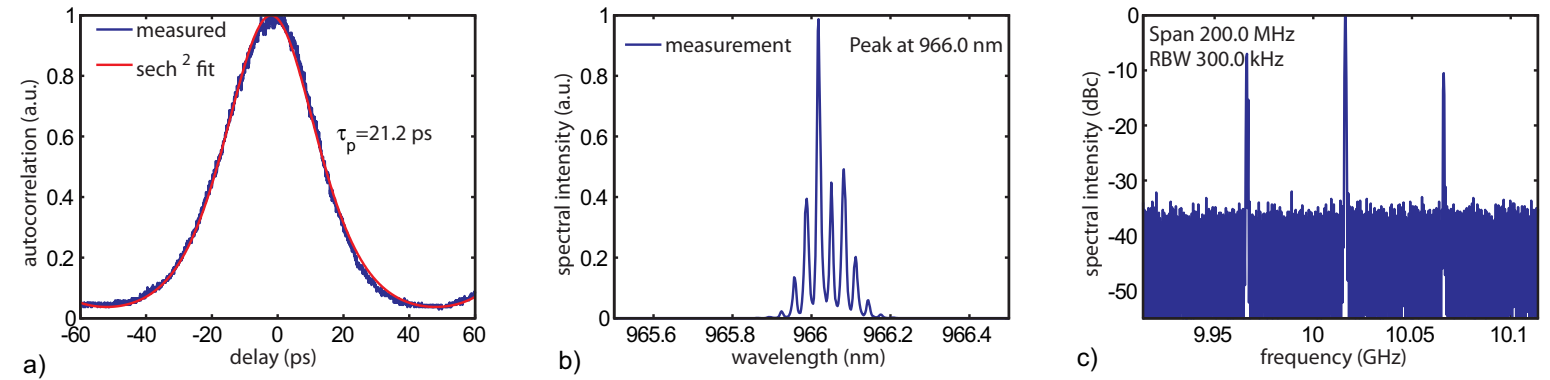

Fig. 2: Measured autocorrelation fitted with an ideal 10-GHz train of 21.2-ps $\operatorname{sech}^{2}$-pulses (a);

Optical spectrum centered at $966 \mathrm{~nm}$ (b); RF-spectrum (c).

\section{Conclusion and outlook}

We demonstrate a 10-GHz MIXSEL with $2.2 \mathrm{~W}$, which is the highest output power from any modelocked 10-GHz semiconductor laser so far. This result was enabled by an optimized antiresonant MIXSEL design with low saturation fluence QD absorbers and with an improved thermal management using a bottom CVD diamond heat spreader. By increasing the mode size on the MIXSEL chip, the average output power can be further increased. Moreover, we envisage the generation of substantially shorter pulses by optimization of the QD absorber's recombination dynamics. The simple MIXSEL geometry should support repetition rates in the $100 \mathrm{GHz}$ regime.

\section{References}

[1] U. Keller, et al., "Semiconductor saturable absorber mirrors (SESAMs) for femtosecond to nanosecond pulse generation in solid-state lasers," IEEE J. Sel. Top. Quantum Electron., vol. 2, pp. 435-453, 1996.

[2] U. Keller, "Recent developments in compact ultrafast lasers," Nature, vol. 424, pp. 831-838, 14.08. 2003.

[3] S. Hoogland, et al., "10-GHz Train of Sub-500-fs Optical Soliton-Like Pulses From a Surface-Emitting Semiconductor Laser," IEEE Phot. Tech. Lett., vol. 17, pp. 267-269, 2005

[4] A. Aschwanden, et al., "10-GHz passively mode-locked surface emitting semiconductor laser with 1.4-W average output power," Appl. Phys. Lett., vol. 86, p. 131102, 2005.

[5] D. Lorenser, et al., "50-GHz passively mode-locked surface-emitting semiconductor laser with $100 \mathrm{~mW}$ average output power," IEEE $J$. Quantum Electron., vol. 42, pp. 838-847, Aug. 2006.

[6] D. J. H. C. Maas, et al., "Vertical integration of ultrafast semiconductor lasers," Appl. Phys. B, vol. 88, pp. 493-497, 2007.

[7] A.-R. Bellancourt, et al., "Modelocked Integrated External-Cavity Surface Emitting Laser (MIXSEL)," IET Optoelectronics, vol. Vol. 3, pp. pp. 61-72, 2009.

[8] B. Rudin, et al., "High-power MIXSEL: an integrated ultrafast semiconductor laser with 6.4 W average power," Accepted for publication in Optics Express, 2010.

[9] J. M. Ostermann, et al., "VCSELs with enhanced single-mode power and stabilized polarization for oxygen sensing," Photonics Technology Letters, IEEE, vol. 17, pp. 2256-2258, 2005. 